\title{
S27. Positive, negative, and affective symptoms in schizophrenia and related psychotic disorders (supported by an educational grant from Janssen B)
}

PRINCIPLE COMPONENTS AND FURTHER POSSIBILITIES WITH THE PANSS.

$L$ von Knorring, $E$ Lindström

Dept. of Psychiatry, University Hospital, S-751 85 Uppsala, Sweden

The Swedish version of the Positive and Negative Syndrome Scale for Schizophrenia (PANSS) has been tested and a satisfactory construct validity and inter-rater reliability has been demonstrated.

In a principal component analysis on a series comprising 120 schizophrenic patients rated by means of the PANSS a Negative, an Excited, a Positive and a Depressive factor were found. These factors were very similar to those earlier described by Kay and Sevy (1990) and Peuskens (1992). However, the Depressive factor included important contributions from anxiety related items and is best explained as an Anxious/Depressive factor. Furthermore, an important Cognitive factor was found. This factor has been seen also in the seven factor results found by Kay and Sevy and Peuskens but has been omitted in the final four factor pyramidical model. However, from our results, it seems reasonable to modify and extend the pyramidical model with a five factor model, including a Cognitive factor. With this five factor model, $70 \%$ of the variance is explained in our principal component analysis.

Later on Kay (1991) introduced the Structured Clinical Interview for the PANSS (SCID-PANSS). It was patterned after other structured interview methods. The structured interview has for a long time been accepted as the most systematic and reliable source of information about symptomatology. The inter-rater reliabilities of the PANSS and the SCID-PANSS were tested in five sessions where video-taped PANSS and SCID-PANSS interviews were independently rated by $17-52$ clinicians. All were experienced clinicians but only a few had scientific training.The inter-rater reliability is increased as compared to the inter-rater reliability achieved by means of the PANSS. As concerns the Positive scale the intra-class coefficients increased to $0.98-0.99$ with the SCID-PANSS. For the Negative scale, the intra-class coefficients increased to $0.83-0.90$ and for the General scale the increase was to $0.95-0.98$ with the SCID-PANSS. Thus, by means of the SCID-PANSS also negative symptoms can be rated with a high inter-rater-reliability.

By means of the five-factor model, it could be demonstrated that risperidone, a selective D-2 and 5-Ht-2 blocker, has effects on all five factors in the schizophrenic syndrome while a traditional neuroleptic such as haloperidol does not influence the cognitive factor significantly.

\section{THE NEGATIVE COMPONENT IN PSYCHOSES}

Professor Dr.med H-J Möller

Universitäts-Nervenklinik und Poliklinik Psychiatrie, Sigmund Freud Straße 25, D-5300 Bonn 1, Germany.

The development and evaluation of neuroleptics in negative symptoms of schizophrenia is becoming more and more of interest. However, severe methodological problems can lead to wrong conclusions in this field. Among other, the differentiation between primary and secondary negative symptoms and the inter-relationship between negative symptoms, depression and akinesia has to be carefully considered. A review of the literature in this field leads to the conclusion that more research efforts are necessary to answer the question whether neuroleptics and which kind of neuroleptics are efficacy in this field. Risperidone, a recently developed $5 \mathrm{HT}_{2}-\mathrm{D}_{2}$ antagonist, seems to be advantageous with respect to negative symptoms. In several studies, among other two large sample multicenter-studies, it proved effective both in positive and minus symptoms. A still ongoing analysis shall answer the question, whether the improvement of negative symptoms is independent from the reduction of positive symptoms. The main results of these studies will be presented. 
NEW PIIARMACOTHERAPEUTIC MODALITIES FOR NEGATIVE SYMPTOMS IN PSYCHOSIS

Jean-Pierre Lindenmayer, MD

Clinical Associate Professor of Psychiatry, Abbert Einstein College of Medicine, USA

Typical antipsychotic drugs have usually been found to have a modest effect on negative symptoms in schizophrenia, although these symptoms are increasingly being recognized as core phenomena of schizophrenia. This new interest has been stimulated by new assessment methods of negative symptoms and by new atypical antipsychotic drugs. These compounds have been reported to have a greater effect on negative symptoms than typical antipsychotics, possibly by way of antagonistic activity at serotonin receptors (5HT2). We will present results of studies of clozapine and risperidone in schizophrenic patients with negative symptoms. First the effects of clozapine will be reviewed in two open trials on positive, negative and affective symptoms as measured by the Postive and Negative Symptom Scale (PANSS) over a 12 month period. Significant effects on negative symptoms are demonstrated. Results of a multidose, placebo-controlled US multicenter trial with risperidone will be presented. Risperidone $6 \mathrm{mg}$ and $16 \mathrm{mg}$ resulted in significant improvement in the PANSS negative subscale as compared with placebo. Haloperidol treated patients did not improve significantly in negative symptoms. These findings suggest that certain atypical antipsychotics may have greater activity against negative symptoms than conventional antipsychotics.

\section{LONG -TERM TREATMENT OF MOOD DISORDERS IN SCHIZOPHRENIA \\ M Azorin \\ Department of Psychiatry, Timone Hospital, 13385 Marseille, France.}

A growing number of studies underline the clinical importance of addressing mood disorders in schizophrenia, due to their frequency, their relationship to poor outcome and risk of relapse and their association with suicide. Johnson ( 1989) has elaborated on four potential ways in which these disorders may be part of the schizophrenic picture : 1 / depression as an ordinary part of the illness, both in the prodromal and acute illness, 2/ postpsychotic depression, 3/ mood disorders in relationship with neuroleptic drugs i.e, true pharmacogenic depression akinetic depression and mood disturbance secondary to tardive syndromes, 4/ schizoaffective illness and related disorders. Recent studies indicate that neuroleptic drugs seem to be the most appropriate treatment for depressed and actively psychotic schizophrenic and schizoaffective patients whereas antidepressants combined with neuroleptics may attenuate and prevent depressive symptoms in postpsychotic schizophrenic and schizoaffective patients (Siris et al 1989). Mood disorders related to neuroleptics may benefit from anticholinergic medication, neuroleptic dose reduction and, in some cases, interruption or change in neuroleptic treatment. There is some evidence that schizoaffective patients and especially those with a predominance of schizomanic episodes and/or affectively ill first degree relatives have a good prophylactic response to mood normalizers. However none of these drugs seems to be superior to neuroleptics in preventing schizoaffective episodes (Taylor 1986). Recently atypical antipsychotics, such as clozapine, have exhibited potential antidepressant and antimanic properties both in affective and schizoaffective patients, some of them having been refractory to classical medications ( Mc Elroy et al 1989 ). These properties seem to be independent of their action on negative symptoms. For risperidone, efficacy on mood-related symptoms has been demonstrated both in short -and long -term treatment (Mertens 1991).Furthermore the incidence of extrapyramidal and tardive syndromes is low with drugs of this kind.For these reasons, atypical antipsychotics appear to be the best candidates for long-term treatment of mood disorders in schizophrenia.
NEW DRUGS FOR THE TREATMENT OF SCIIZOPHRENIC PATIENTS

W W Fleischhacker

Dept. of Biological Psychiatry, University of Innsbruck, Medical School, Anichstrasse 35, A-6020 Innsbruck, Austria

Following the breakthrough in the treatment of schizophrenia that was initiated by the introduction of antipsychotic drugs in the 1950's the field has been eager to improve these drugs in terms of efficacy and side effect profiles. Progress was slow for decades and various reveiws in the eighties correctly stated that clinical trials did not really show advantages of any one neuroleptic over any other. Clozapine, the development of which was hampered by its propensity to cause agranulocytosis led the way in the search for new antipsychotics. The increasing understanding of its pharmacological actions as well as clinical trials that showed superior efficacy over classical antipsychotics stimulated new theories about the pathophysiology of schizophrenia as well as the development of new drugs A list of antipsychotics, that have either become available recently or are at least in phase III includes risperidone and zotepine as well as olanzapine, seroquel and sertindole. These drugs will be discussed in respect of their effects on the positive symptoms of schizophrenia. 